\title{
Effect of atherothrombotic aorta on outcomes of total aortic arch replacement
}

Kenji Okada, MD, PhD, Atsushi Omura, MD, Hiroya Kano, CE, Takeshi Inoue, MD, Takanori Oka, MD, Hitoshi Minami, MD, $\mathrm{PhD}$, and Yutaka Okita, $\mathrm{MD}, \mathrm{PhD}$

Objective: The effect of an atherothrombotic aorta on the short- and long-term outcomes of total aortic arch replacement, including postoperative neurologic deficits, remains unknown. We evaluated this relationship and also elucidated the synergistic effect of multiple other risk factors, in addition to an atherothrombotic aorta, on the neurologic outcome.

\begin{abstract}
Methods: A group of 179 consecutive patients undergoing total aortic arch replacement were studied. An atherothrombotic aorta was present in 34 patients (19\%), more than moderate leukoaraiosis in $71(39.7 \%)$, and significant extracranial carotid artery stenosis in $27(15.1 \%)$. In-hospital deaths occurred in 2 patients, $1(2.9 \%)$ of 34 patients with and $1(0.7 \%)$ of 145 patients without an atherothrombotic aorta $(P=.26)$. Permanent neurologic deficits occurred in $4(2.2 \%)$ and transient neurologic deficits in $17(9.5 \%)$ patients. Multivariate analysis demonstrated that the risk factors for transient neurologic deficits were an atherothrombotic aorta (odds ratio, 4.4), extracranial carotid artery stenosis (odds ratio, 5.5), moderate/severe leukoaraiosis (odds ratio, 3.6), and cardiopulmonary bypass time (odds ratio, 1.02). To calculate the probability of transient neurologic deficits, the following equation was derived: probability of transient neurologic deficits $=\{1+\exp [7.276-1.489$ (atherothrombotic aorta) - 1.285 (leukoaraiosis) - 1.701 (extracranial carotid artery stenosis) -0.017 (cardiopulmonary bypass time) $]\}^{-1}$. An exponential increase occurred in the probability of transient neurologic deficits with presence of an atherothrombotic aorta and other risk factors in relation to the cardiopulmonary bypass time. Survival at 3 years after surgery was significantly reduced in patients with vs without an atherothrombotic aorta $(75.0 \% \pm 8.8 \%$ vs $89.2 \% \pm 3.1 \%, P=.01)$.
\end{abstract}

Conclusions: Patients with an atherothrombotic aorta and associated preoperative comorbidities might be predisposed to adverse short- and long-term outcomes, including transient neurologic deficits. (J Thorac Cardiovasc Surg 2013;145:984-91)

Supplemental material is available online.

Although the short- and long-term outcomes of total aortic arch replacement (TAR) have been improving during the past decade, ${ }^{1-3}$ the procedure is likely to be associated with life-threatening complications, such as neurologic deficits, pulmonary dysfunction, and bowel ischemia/necrosis. Because postoperative neurologic deficits have been reported to adversely affect the short- and long-term outcomes, ${ }^{4,5}$ it is crucial to identify the risk factors and prevent complications to the extent possible. Atherosclerotic disease of the aortic arch itself has been

\footnotetext{
From the Division of Cardiovascular Surgery, Department of Surgery, Kobe University Graduate School of Medicine, Kobe, Japan.

Disclosures: Authors have nothing to disclose with regard to commercial support.

Received for publication Jan 20, 2012; revisions received March 1, 2012; accepted for publication March 19, 2012; available ahead of print May 10, 2012.

Address for reprints: Yutaka Okita, MD, PhD, Division of Cardiovascular Surgery, Department of Surgery, Kobe University Graduate School of Medicine, 7-5-2, Kusunoki-Cho, Chuo-Ku, Kobe, Hyogo 650-0017, Japan (E-mail: yokita@med. kobe-u.ac.jp).

$0022-5223 / \$ 36.00$

Copyright (C) 2013 by The American Association for Thoracic Surgery doi:10.1016/j.jtcvs.2012.03.048
}

historically reported by Amarenco and associates ${ }^{6}$ to be a potential source of cerebral emboli. "Shaggy aorta," a term advocated by Hollier and associates, ${ }^{7}$ was defined as very extensive atheromatous disease with diffuse ulcers associated with soft, loosely held debris and a paucity of actual thrombus, now recognized as an atherothrombotic aorta, a pathologic entity. Recent advances in contrast-enhanced computed tomography (CT) have allowed accurate preoperative imaging of the atherothrombotic aorta. However, to date, no reports have specifically studied the effect of an atherothrombotic aorta on the short- and long-term outcomes of TAR, including postoperative neurologic deficits.

Neurologic deficits can be caused not only by embolic events such as those resulting from the presence of an atherothrombotic aorta, but also by inadequate perfusion during cardiopulmonary bypass (CPB). Cerebrovascular disease, including leukoaraiosis and carotid artery stenosis/occlusion, are also believed to augment ischemic events during CPB. Leukoaraiosis is defined as patchy punctuate or confluent hyperintensity in the white matter and deep gray nuclei on $\mathrm{T}_{2}$-weighed brain magnetic resonance imaging (MRI), reflecting chronic ischemic changes in the myelin and axons. ${ }^{8}$ In the current era of an aging population, the number of patients with the combination of an 


\section{Abbreviations and Acronyms \\ $\mathrm{CPB}=$ cardiopulmonary bypass \\ CT $\quad=$ computed tomography \\ euroSCORE $=$ European System for Cardiac \\ Operative Risk Evaluation

$\begin{array}{ll}\text { MRA } & =\text { magnetic resonance angiography } \\ \text { MRI } & =\text { magnetic resonance imaging } \\ \text { OR } & =\text { odds ratio } \\ \text { PND } & =\text { permanent neurologic deficit } \\ \mathrm{P}_{\text {TND }} & =\text { probability of TNDs } \\ \text { RCP } & =\text { retrograde cerebral perfusion } \\ \text { SCP } & =\text { selective cerebral perfusion } \\ \text { TAR } & =\text { total aortic arch replacement } \\ \text { TND } & =\text { transient neurologic deficit }\end{array}$

atherothrombotic aorta, leukoaraiosis, and carotid artery disease is increasing. Accordingly, it has become important to determine whether there are synergistic adverse effects of these multiple risk factors on the neurologic outcome after TAR. Therefore, we performed the present retrospective study to investigate the effect of an atherothrombotic aorta and associated risk factors on the short- and long-term outcomes after TAR.

\section{METHODS}

Our institutional review board approved the present study, and the need for individual consent was waived. We retrospectively studied 179 consecutive patients with a mean age of $73.8 \pm 7.7$ years who had undergone TAR through a median sternotomy from January 2002 to December 2010. All patients at our institute routinely undergo MRI/magnetic resonance angiography (MRA), duplex ultrasonography, and CT before undergoing elective aortic surgery. According to the concepts formulated by Hollier and colleagues ${ }^{7}$ and Amarenco and colleagues, ${ }^{6}$ when fragile and spiculated atheroma that exceeded $5 \mathrm{~mm}$ in the thickness was confirmed by CT in the ascending aorta or aortic arch but excluding the aneurysm itself, an "atherothrombotic aorta" was diagnosed (Figure 1). In the present series, an atherothrombotic aorta was present in the ascending or aortic arch in $34 \mathrm{pa}-$ tients $(19.0 \%)$. Patients with an atherothrombotic aorta were compared with 145 patients without an atherothrombotic aorta with regard to preoperative patient characteristics, intraoperative variables, and short- and longterm outcomes.

\section{Neuromagnetic Imaging}

Brain MRI was performed using a 1.5-T scanner. A standardized imaging protocol was used, consisting of axial $\mathrm{T}_{1}$-weighted, $\mathrm{T}_{2}$-weighted, and fast fluid attenuated inversion recovery images. The intracranial and extracranial vasculature was evaluated preoperatively using MRA. All patients underwent routine 2-dimesional time of flight MRA through the neck and 3-dimensional time of flight MRA through the circle of Willis. Postoperative cerebral infarction or ischemia was detected using diffusion-weightedMRI or brain CT, when patients had any symptoms related to transient neurologic deficits (TNDs) or permanent neurologic deficits (PNDs).

\section{Leukoaraiosis Rating}

Leukoaraiosis was rated using the Scheltens scale. ${ }^{9}$ Each cerebral region was initially scored for lesion size and then for lesion number. In accordance with this scale, the periventricular white matter hyperintensities were scored on 3 regions: the frontal and occipital caps and the periventricular bands. They were rated as none, score $0 ; 5 \mathrm{~mm}$ or less, score 1; and confluent lesions and those greater than $5 \mathrm{~mm}$, score 2 . The deep white matter hyperintensities were examined in 4 subcortical regions (frontal, parietal, temporal, and occipital lobes). These lesions were rated as none, score $0 ; 3 \mathrm{~mm}$ and smaller and 5 or fewer lesions, score $1 ; 3 \mathrm{~mm}$ or smaller and 6 or more lesions, score $2 ; 4$ to $10 \mathrm{~mm}$ and 5 or fewer lesions, score $3 ; 4$ to 10 $\mathrm{mm}$ and 6 or more lesions, score $4 ; 10 \mathrm{~mm}$ or larger and 1 or more lesions, score 5; and large confluent lesions, score 6 . The total leukoaraiosis score is the sum of the periventricular white matter intensity and deep white matter hyperintensity subscores, with a maximum score of 30 . A score greater than 10 was defined as more than moderate leukoaraiosis.

\section{Evaluation of Intracranial Carotid Artery by MRA}

The severity of the involvement of the intracranial vasculature and the circle of Willis was evaluated by MRA. Because precise evaluation of the degree of stenosis was difficult, the occlusion of 1 of the intracranial vessels (ie, intracranial carotid artery; anterior, middle, or posterior cerebral artery; basilar artery; or the circle of Willis) was considered indicative of the presence of significant intracranial vascular disease.

The evaluation of the extracranial carotid artery focused on the common carotid artery, which was evaluated by both MRA and duplex ultrasonography. Carotid stenosis was defined as the presence of stenosis of greater than $70 \%$, combined with the demonstration of a peak systolic velocity greater than $250 \mathrm{~cm} / \mathrm{s}$ on duplex ultrasound imaging using the North American Symptomatic Carotid Endarterectomy method. ${ }^{10}$

The patient characteristics are listed in Table 1 . Of the 179 patients, 174 $(97.2 \%)$ underwent TAR on an elective basis. No significant differences were found between patients with and without an atherothrombotic aorta in age, gender, diabetes mellitus, or chronic kidney disease (creatinine $\geq 2.0 \mathrm{mg} / \mathrm{dL}$ ), although significant differences were found in coronary artery disease, left ventricular dysfunction (ejection fraction $\leq 40 \%$ ), and chronic obstructive pulmonary disease. Regarding preoperative cerebrovascular disease, no significant difference was found between the 2 groups in the history of cerebral infarction, intracranial or extracranial carotid artery disease, or leukoaraiosis. Significant differences were present between patients with and without an atherothrombotic aorta in the additive European System for Cardiac Operative Risk Evaluation (euroSCORE) $(8.6 \pm 1.9$ vs $7.6 \pm 2.0$, respectively; $P=.009)$, logistic euroSCORE $(16.1 \pm 8.9$ vs $12.2 \pm 7.0$, respectively; $P=0.007)$, and Japan score ${ }^{11}$ $(6.3 \pm 4.2$ vs $4.8 \pm 2.5$, respectively; $P=.01)$, indicating that patients with an atherothrombotic aorta were at greater risk.

\section{Surgical Protocol}

The details of TAR at our institute have been previously reported. ${ }^{3}$ The cannulation site and type of arterial cannulation for CPB is particularly important. Both transesophageal and periaortic echocardiography were applied to interrogate the ascending aorta and determine the proper cannulation site. A 24F dispersion arterial cannula (Duraflo II; Edwards Lifesciences, Irvine, Calif) was inserted near the aortic root with perfusion toward the aortic valve in $85.3 \%$ of patients with an atherothrombotic aorta $(P=.0002$ vs without an atherothrombotic aorta). After the tympanic temperature had decreased to $20^{\circ}$ to $23^{\circ} \mathrm{C}$ and the rectal temperature to less than $30^{\circ} \mathrm{C}$, the aortic arch aneurysm was opened, and retrograde cerebral perfusion $(\mathrm{RCP} ; \mathrm{n}=2)$ or antegrade selective cerebral perfusion (SCP; $\mathrm{n}=177)$ was instituted. RCP was applied for 2 patients with mobile plaques in their common carotid arteries. If the ostium of the arch vessels were severely atherosclerotic, arteriotomy of the arch vessels was extended from the diseased ostium to a relatively clear distal part and then cannulas were placed in position under direct vision. The distal aortic arch or descending aorta was divided from inside the aorta to avoid injury to the recurrent nerve during circulatory arrest to the lower body. A gelatin-impregnated quadfurcated Dacron graft (Gelweave, Vascutek, Terumo, Scotland, UK; J Graft Shield Neo, Japan Lifeline, Tokyo, Japan) or Triplex (Terumo, Tokyo, Japan) 


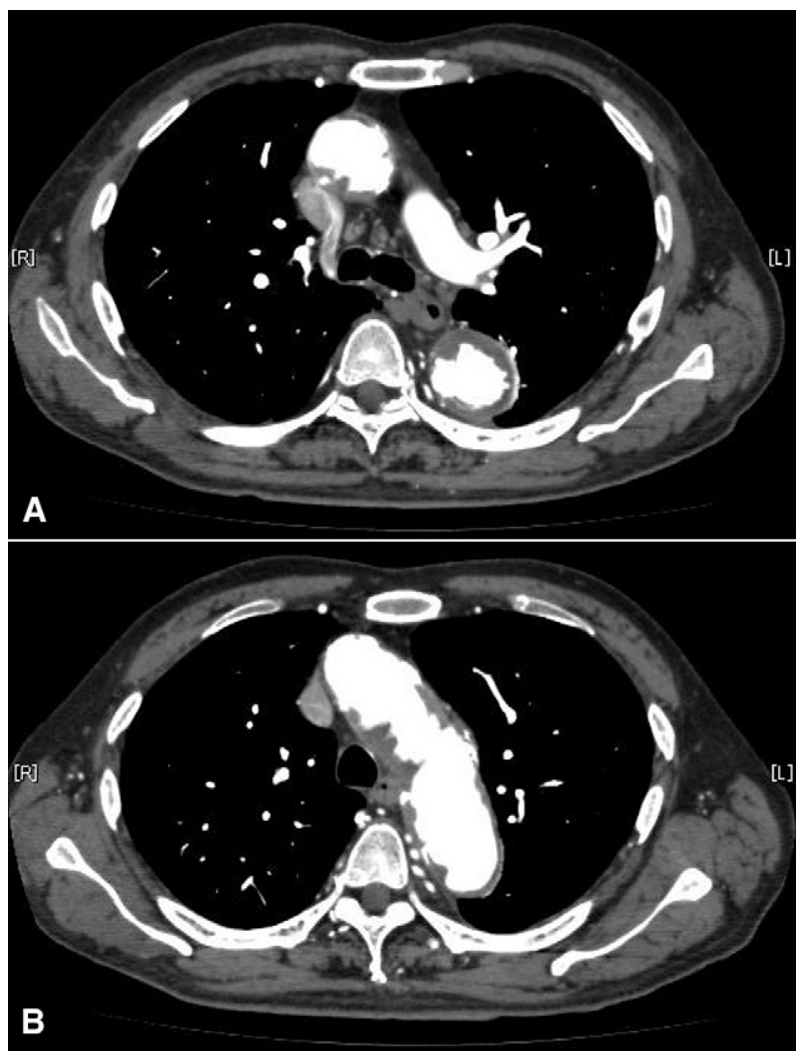

FIGURE 1. Contrast-enhanced computed tomography $(C T)$ scans demonstrating representative findings of atherothrombotic aorta in both (A) ascending aorta and (B) aortic arch. Very thick $(9 \mathrm{~mm})$ and spiculated atheroma observed in both parts of the aortic wall.

was used. After completion of the distal anastomosis of the prosthetic graft, lower body circulation was reinstituted thorough a branch graft, and the tympanic/rectal temperature was rewarmed early to $33^{\circ} \mathrm{C}$ to shorten the CPB time. The proximal anastomosis of the graft was completed, followed by coronary reperfusion. Finally, the aortic arch vessels were reconstructed. If patients had significant carotid artery stenosis, leukoaraiosis, and/or history of symptomatic old cerebral infarction, we reattached the arch vessels without rewarming after distal anastomosis of the graft $(n=16)$.

\section{Definition of Neurologic Deficits}

Using the definition of the Mount Sinai group ${ }^{4}$ PNDs were defined as the presence of deficits that persisted at hospital discharge. TNDs were defined as delayed awakening, transient loss of orientation, slurred language, agitation, poor response to commands, and transient hemiparesis that had recovered by hospital discharge.

\section{Statistical Analysis}

The data were processed using SPSS, version 11.0, software (SPSS, Chicago, Ill). Continuous values are expressed as the mean \pm standard deviation and were compared using $t$-tests. Data were analyzed using the chisquare test for categorical variables. Stepwise logistic regression analysis was performed to identify the risk factors for PNDs and TNDs. Clinically relevant variables with $P<.05$ on univariate analysis were incorporated into the multivariate models. Multivariate logistic regression models were developed to identify significant independent factors. The final models were converted to probability estimates through the equation $\mathrm{P}=\{1+\exp -(\mathrm{A}+\mathrm{BX})\}^{-1}$, where $\mathrm{X}$ is a vector of risk factor values, $\mathrm{A}$ is the estimated constant, and B is a vector of estimated coefficients. Also, 95\% confidence intervals (CIs) for the estimated probabilities were constructed on the basis of the estimated covariate matrix. Survival was assessed using the Kaplan-Meier method, and a log-rank test was applied for comparison.

\section{RESULTS}

The average CPB time, myocardial ischemic time, lower body circulatory arrest time, and antegrade SCP time was $164.4 \pm 44.3$ minutes, $69.8 \pm 28.2$ minutes, $38.5 \pm 12.3$ minutes, and $87.4 \pm 21.4$ minutes, respectively (Table 1 ). The average CPB time and lower body circulatory arrest time were significantly longer in patients with vs without an atherothrombotic aorta.

The 30-day mortality was $0 \%$ and the hospital mortality was $1.1 \%(2 / 179)$. The cause of death was bowel necrosis in 1 patient with an atherothrombotic aorta and pulmonary dysfunction in 1 patient without an atherothrombotic aorta, for a mortality of $2.9 \%$ in the former group and $0.7 \%$ in the latter group $(P=.26)$. Overall, PNDs occurred in 4 patients $(2.2 \%)$. The details concerning these 4 patients are listed in Table E1. All had leukoaraiosis, but none had extracranial carotid artery stenosis. Postoperative brain imaging demonstrated multiple embolizations in 3 of the 4 patients. Dispersion cannulas were not used for patients 2, 3 and 4 . Patient 1 had a history of stroke with hemiplegia, and postoperative brain imaging demonstrated aggravation of a pre-existing infarction. The average CPB time for the 4 patients was $239.8 \pm 87.6$ minutes. Univariate analysis demonstrated that the risk factors for PNDs were chronic kidney disease (odds ratio [OR], 11.5, 95\% CI, 1.5-88.0, $P=.026$ ) and CPB time (OR, 1.02; 95\% CI, 1.01-1.04; $P=0.0005$ ). However, multivariate analysis demonstrated a correlation only with $\mathrm{CPB}$ time (OR, 1.02; 95\% CI, 1.01-1.04; $P=.02)$.

TNDs occurred in 17 patients $(9.5 \%)$ and was present in $9(26.5 \%)$ of the 34 patients with and $8(5.5 \%)$ of the 145 without an atherothrombotic aorta $(P=.0002)$. Additional details concerning the TNDs in these 17 patients are listed in Table 2. Of these 17 patients, $9(52.9 \%)$ had an atherothrombotic aorta, $8(47.1 \%)$ had extracranial carotid artery stenosis, and $12(70.6 \%)$ had leukoaraiosis. The major symptoms of TNDs were delirium in 8 , delayed awakening in 4, and transient hemiparesis in 3. Postoperative diffusionweighted MRI demonstrated multiple embolizations in 5 of the 17 patients. Dispersion cannulas were used for 16 of the 17 patients, and the average CPB time was $205.6 \pm 51.2$ minutes. The univariate and multivariate analysis results for TNDs are listed in Table 3. On multivariate analysis, the risk factors for TNDs were atherothrombotic aorta (OR, 4.4; $P=.026$ ), extracranial carotid artery stenosis (OR, 5.5; $P=.01$ ), more than moderate leukoaraiosis (OR, 3.6; $P=.05$ ), and CPB time (OR, 1.02; $P=.008)$.

To calculate the probability of TNDs $\left(\mathrm{P}_{\mathrm{TND}}\right)$, the following equation was derived (enter 1 if a factor is present, 0 if 
TABLE 1. Patient characteristics

\begin{tabular}{|c|c|c|c|c|}
\hline \multirow[b]{2}{*}{ Variable } & \multirow[b]{2}{*}{ Total } & \multicolumn{2}{|c|}{ Atherothrombosis } & \multirow{2}{*}{$\begin{array}{c}P \\
\text { value } \\
\end{array}$} \\
\hline & & Yes & No & \\
\hline Patients (n) & 179 & 34 & 145 & \\
\hline Age $(y)$ & $73.8 \pm 7.7$ & $74.4 \pm 6.1$ & $73.6 \pm 8.1$ & .61 \\
\hline Octogenarians (n) & 37 (20.7) & 7 (20.6) & $30(20.7)$ & .99 \\
\hline Male gender & $147(82.1)$ & $31(91.2)$ & $116(80.0)$ & .13 \\
\hline Elective & $174(97.2)$ & $33(97.1)$ & $141(97.2)$ & .95 \\
\hline Diabetes mellitus & $30(16.8)$ & $8(23.5)$ & $22(15.2)$ & .24 \\
\hline Hypertension & $160(89.4)$ & $30(88.2)$ & $130(89.7)$ & .81 \\
\hline Dyslipidemia & $76(42.5)$ & $16(47.1)$ & $60(41.4)$ & .55 \\
\hline Smoking & $134(74.9)$ & $27(79.4)$ & $107(73.8)$ & .50 \\
\hline Old cerebral infarction & $17(9.5)$ & $6(17.6)$ & $11(7.6)$ & .07 \\
\hline Coronary artery disease & $56(31.3)$ & $18(52.9)$ & $38(26.2)$ & $.0025^{*}$ \\
\hline Left ventricular ejection fraction $(\leq 40 \%)$ & $8(4.5)$ & $4(11.8)$ & $4(2.8)$ & $.022 *$ \\
\hline Chronic obstructive pulmonary disease & $20(11.2)$ & 7 (20.6) & $13(9.0)$ & $.05^{*}$ \\
\hline Chronic kidney disease (creatinine $\geq 2.0 \mathrm{mg} / \mathrm{dL}$ ) & $16(8.9)$ & $3(8.8)$ & $13(9.0)$ & .98 \\
\hline \multicolumn{5}{|l|}{ Cerebrovascular disease } \\
\hline Extracranial carotid artery stenosis & $27(15.1)$ & $8(23.5)$ & $19(13.1)$ & .13 \\
\hline Intracranial vascular occlusion & $15(8.4)$ & $4(11.8)$ & $11(7.6)$ & .43 \\
\hline Leukoaraiosis score & $8.4 \pm 5.9$ & $9.5 \pm 6.2$ & $8.1 \pm 5.9$ & .22 \\
\hline More than moderate leukoaraiosis & $71(39.7)$ & $16(47.1)$ & 55 (37.9) & .33 \\
\hline euroSCORE & $7.8 \pm 2.0$ & $8.6 \pm 1.9$ & $7.6 \pm 2.0$ & $.009 *$ \\
\hline Logistic euroSCORE & $12.9 \pm 7.5$ & $16.1 \pm 8.9$ & $12.2 \pm 7.0$ & $.007 *$ \\
\hline Japan score (30-day mortality) & $5.1 \pm 2.9$ & $6.3 \pm 4.2$ & $4.8 \pm 2.5$ & $.01 *$ \\
\hline Japan score/complications & $21.9 \pm 9.6$ & $25.9 \pm 9.6$ & $21.0 \pm 9.4$ & $.007 *$ \\
\hline Concurrent procedure & $78(43.6)$ & $21(61.8)$ & $57(39.3)$ & $.018^{*}$ \\
\hline \multicolumn{5}{|l|}{ Cannulation sites/type for CPB } \\
\hline Ascending aorta/arch & $171(95.5)$ & $31(91.2)$ & $140(96.6)$ & .17 \\
\hline 24F dispersion cannula & $102(57.0)$ & $29(85.3)$ & $73(50.3)$ & $.0002 *$ \\
\hline ASCP & $177(98.9)$ & $34(100)$ & 143 (98.6) & .49 \\
\hline DHCA/RCP & $2(1.1)$ & $0(0)$ & $2(1.4)$ & .49 \\
\hline CPB time (min) & $164.4 \pm 44.3$ & $179.1 \pm 48.4$ & $161.0 \pm 42.3$ & $.03 *$ \\
\hline Myocardial ischemic time (min) & $69.8 \pm 28.2$ & $77.9 \pm 30.1$ & $67.9 \pm 27.5$ & .06 \\
\hline Lower body DHCA time (min) & $38.5 \pm 12.3$ & $42.4 \pm 11.4$ & $37.6 \pm 12.3$ & $.04 *$ \\
\hline ASCP time (min) & $87.4 \pm 21.4$ & $89.4 \pm 23.0$ & $86.9 \pm 21.1$ & .5 \\
\hline Minimal tympanic temperature $\left({ }^{\circ} \mathrm{C}\right)$ & $21.5 \pm 1.6$ & $21.5 \pm 1.4$ & $21.5 \pm 1.6$ & .99 \\
\hline Minimal rectal temperature $\left({ }^{\circ} \mathrm{C}\right)$ & $26.1 \pm 2.0$ & $26.0 \pm 1.8$ & $26.1 \pm 2.1$ & .7 \\
\hline
\end{tabular}

Data presented as mean \pm SD or numbers, with percentages in parentheses. euroSCORE, European System for Cardiac Operative Risk Evaluation; $C P B$, cardiopulmonary bypass; $A S C P$, antegrade selective cerebral perfusion; $D H C A$, deep hypothermic circulatory arrest; $R C P$, retrograde cerebral perfusion. *Significantly different at $P<.05$ with and without a shaggy aorta.

absent, and CPB time in minutes): $\mathrm{P}_{\mathrm{TND}}=\{1+\exp [7.276-$ 1.489 (atherothrombotic aorta) - 1.285 (leukoaraiosis) 1.701 (extracranial carotid artery stenosis) -0.017 (CPB time) $]\}^{-1}$. The logistic curves showing the probability of TND with the different predisposing factors are shown in Figure 2, which displays an exponential increase in the probability with compounding of the different risk factors as the $\mathrm{CPB}$ time increased. The $\mathrm{P}_{\mathrm{TND}}$ at 160 minutes (our average $\mathrm{CPB}$ time) in patients without any risks (null) was $1.0 \%$; with an atherothrombotic aorta, it was $4.4 \%$; with an atherothrombotic aorta and leukoaraiosis, $14.4 \%$; and with an atherothrombotic aorta, leukoaraiosis, and extracranial carotid artery disease, $48.0 \%$.

Additional postoperative complications are listed in Table E2. Patients with an atherothrombotic aorta had significantly more morbidities of paraparesis, prolonged ventilation ( $\geq 48$ hours), and bowel necrosis.

Long-term follow-up was complete for $94.4 \%$ of the patients, and the mean follow-up period was $2.8 \pm 2.3$ years. Figure 3 shows the overall long-term survival assessed using the Kaplan-Meier method. Survival at 3 years after surgery was significantly reduced in the patients with vs without an atherothrombotic aorta $(75.0 \% \pm 8.8 \%$ and $89.2 \% \pm 3.1 \%$, respectively; $P=.01$ ).

\section{DISCUSSION}

Early mortality has been greatly improved in contemporary TAR, as shown in recent studies. ${ }^{1-3}$ Our present study has demonstrated that the overall 30-day mortality was $0 \%$ and hospital mortality was $1.1 \%$. However, TAR is 
TABLE 2. Details of TNDs

\begin{tabular}{|c|c|c|c|c|c|c|c|c|}
\hline Pt. no. & Age $(y)$ & Symptoms & $\begin{array}{c}\text { Postoperative } \\
\text { MRI/CT findings }\end{array}$ & A & $\mathbf{E}$ & $\mathbf{L}$ & $\mathbf{O}$ & CPB time (min) \\
\hline 1 & 77 & Delirium & NA & No & No & Yes & No & 154 \\
\hline 2 & 84 & Delayed awakening & Null & No & No & No & No & 224 \\
\hline 3 & 77 & Delayed awakening & Solitary & Yes & No & No & No & 223 \\
\hline 4 & 79 & Hemiparesis, convulsion & Solitary & No & Yes & Yes & No & 227 \\
\hline 5 & 75 & Delirium & NA & Yes & Yes & Yes & No & 143 \\
\hline 6 & 75 & Right arm numbness & Multiple & No & No & Yes & No & 132 \\
\hline 7 & 70 & Delirium & Null & No & Yes & Yes & Yes & 126 \\
\hline 8 & 74 & Delayed awakening & Null & No & Yes & Yes & Yes & 224 \\
\hline 9 & 74 & Delirium & Null & Yes & No & Yes & No & 216 \\
\hline 10 & 67 & Delirium & Null & Yes & Yes & No & No & 208 \\
\hline 11 & 81 & Delirium & NA & Yes & No & No & No & 289 \\
\hline 12 & 75 & Delayed awakening & Null & Yes & No & Yes & No & 222 \\
\hline 13 & 75 & Convulsion & Multiple & Yes & Yes & Yes & No & 251 \\
\hline 14 & 77 & Hemiparesis & Null & No & No & Yes & No & 243 \\
\hline 15 & 65 & Hemiparesis & Multiple & Yes & No & Yes & Yes & 288 \\
\hline 16 & 72 & Delirium & Multiple & Yes & Yes & Yes & No & 141 \\
\hline 17 & 82 & Delirium & Multiple & No & Yes & No & No & 184 \\
\hline
\end{tabular}

TNDs, Transient neurologic deficits; Pt. no., patient number; MRI, magnetic resonance imaging; $C T$, computed tomography; $A$, atherothrombotic aorta; $E$, extracranial carotid artery stenosis; $L$, leukoaraiosis; $O$, old cerebral infarction; $C P B$, cardiopulmonary bypass; $N A$, not applicable.

likely to be associated with life-threatening complications, such as neurologic deficits, pulmonary dysfunction, and bowel ischemia/necrosis. Particularly relevant to both short- and long-term outcomes in TAR are neurologic deficits. ${ }^{4,5}$ Although the reported overall incidence of PNDs and TNDs ranging from $0 \%$ to $4.4 \%$ and $2.5 \%$ to $9.9 \%$ appears to be favorable, a subgroup of patients still exists that is at high risk of postoperative neurologic deficits, regardless of the method of brain protection used during aortic arch surgery. ${ }^{1-3,12,13}$ As noted, with the aging of society, TAR is likely to be performed more frequently in patients with an increased prevalence of cerebrovascular disease and severe atheromatous disease in the ascending aorta or aortic arch, increasing the incidence of PNDs and TNDs in the elderly. Identifying the risk factors that could adversely affect neurologic outcomes is potentially important in reducing the neurologic morbidity and mortality of TAR.

A number of risk factors for PNDs and TNDs in TAR, including female gender, diabetes mellitus, history of cerebrovascular accident, and severe atheromatous changes, have been identified in the published data. Although aortic pathology has not been included in preoperative risk stratification systems such as euroSCORE, it is generally

TABLE 3. Risk factors for TNDs identified on univariate and multivariate analyses

\begin{tabular}{|c|c|c|c|c|}
\hline \multirow[b]{2}{*}{ Variable } & \multicolumn{2}{|c|}{ Univariate } & \multicolumn{2}{|c|}{ Multivariate } \\
\hline & OR $(95 \%$ CI $)$ & $P$ value & OR $(95 \%$ CI $)$ & $P$ value \\
\hline Octogenarian & $0.81(0.22-2.97)$ & .75 & & \\
\hline Male gender & $1.71(0.37-7.86)$ & .49 & & \\
\hline Atherothrombotic aorta & $6.17(2.17-17.5)$ & .0006 & $4.43(1.19-16.5)$ & .026 \\
\hline Extracranial carotid artery stenosis & $6.70(2.30-19.4)$ & .0005 & $5.48(1.50-20.1)$ & .01 \\
\hline Intracranial vasculature occlusion & $1.53(0.32-7.43)$ & .60 & & \\
\hline More than moderate leukoaraiosis & $4.19(1.41-12.5)$ & .01 & $3.62(1.00-13.6)$ & .05 \\
\hline Diabetes mellitus & $0.64(0.14-2.95)$ & .57 & & \\
\hline Old cerebral infarction & $2.27(0.58-8.85)$ & .24 & & \\
\hline Coronary artery disease & $3.60(1.29-10.0)$ & .014 & $0.88(0.20-3.84)$ & .87 \\
\hline Low LVEF $(\leq 40 \%)$ & $12.2(2.72-54.3)$ & .001 & $5.67(0.83-38.5)$ & .08 \\
\hline COPD & $4.08(1.27-13.2)$ & .019 & $2.57(0.48-13.9)$ & .27 \\
\hline CKD (creatinine $\geq 2.0 \mathrm{mg} / \mathrm{dL}$ ) & $2.46(0.62-9.67)$ & .20 & & \\
\hline CPB time & $1.01(1.01-1.03)$ & .0004 & $1.02(1.00-1.03)$ & .008 \\
\hline ASCP time (min) & $1.00(0.98-1.03)$ & .89 & & \\
\hline Minimal tympanic temperature $\left({ }^{\circ} \mathrm{C}\right)$ & $0.72(0.51-1.02)$ & .07 & & \\
\hline
\end{tabular}

$T N D s$, Transient neurologic deficits; $O R$, odds ratio; $C I$, confidence interval; $L V E F$, left ventricular ejection fraction; $C O P D$, chronic obstructive pulmonary disease; $C K D$, chronic kidney disease; $C P B$, cardiopulmonary bypass; $A S C P$, antegrade selective cerebral perfusion. 


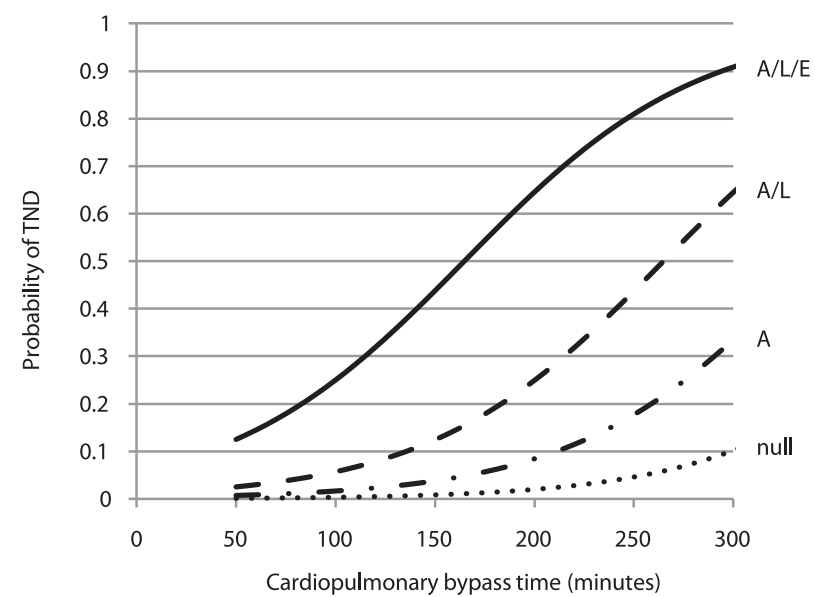

FIGURE 2. Logistic curve of the probability of transient neurologic deficits $(T N D s)$ in relation to cardiopulmonary bypass $(C P B)$ time with varying combinations of 3 risk factors (atherothrombotic aorta, leukoaraiosis, extracranial carotid artery disease). Null, Without any risks; $A$, atherothrombotic aorta; $A / L$, atherothrombotic aorta and leukoaraiosis; $A / L / E$, atherothrombotic aorta, leukoaraiosis, and extracranial carotid artery stenosis.

suspected to be one of the risk factors for adverse neurologic outcomes. A shaggy aorta is the prototypical potential embolic source for neurologic deficits and was defined by Hollier and associates. ${ }^{7}$ From the findings by Amarenco and associates ${ }^{6}$ of a critical threshold of $4 \mathrm{~mm}$ in thickness of atherosclerotic plaque, for our study, we defined an atherothrombotic aorta as being present when fragile and spiculated atheroma greater than $5 \mathrm{~mm}$ in thickness was confirmed by both CT and visual inspection of the ascending aorta or aortic arch. Previously, the effect of an atherothrombotic aorta on the morbidities and, in particular, on neurologic deficits and short- and long-term outcomes has remained largely unknown. However, in our study, we have demonstrated that patients with an atherothrombotic aorta showed significantly greater additive/logistic euroSCORE and Japan scores because of coronary artery disease, impaired left ventricular function, and chronic obstructive pulmonary disease, all factors universally recognized as adversely affecting short- and long-term

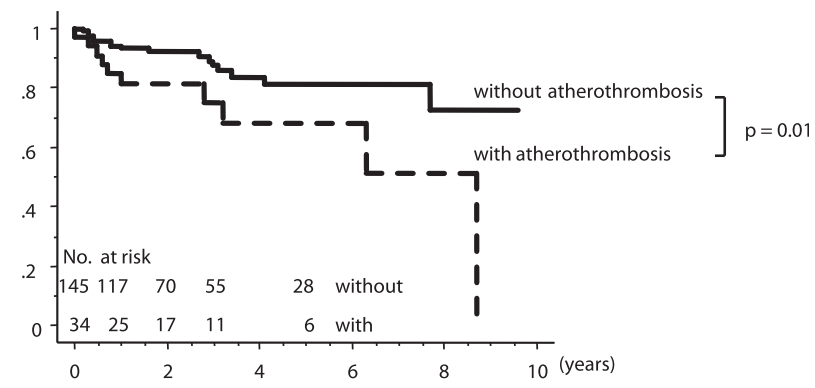

FIGURE 3. Survival curve for patients with (dashed line) and without (solid line) atherothrombotic aorta showing survival at 3 years after surgery of $75.0 \% \pm 8.8 \%$ and $89.2 \% \pm 3.1 \%(P=.01)$, respectively. outcomes. Contrary to our expectations, no significant difference in the prevalence of diabetes mellitus, hypertension, dyslipidemia, and smoking was found in the patients with vs without an atherothrombotic aorta.

With regard to operative details, $24 \mathrm{~F}$ dispersion cannulas were used more often in patients with an atherothrombotic aorta and were used in $85.3 \%$ of patients in this group. This was in accordance with the findings from Fukuda and associates, ${ }^{14}$ who demonstrated that directing the cannula tip of the dispersion cannula toward the aortic root generated slower and less turbulent flow in the transverse arch of the glass models of both healthy and aneurysmal aortic arches. Right axillary artery perfusion is one of the useful options for arterial cannulation, and its efficacy has been reported. ${ }^{2}$ However, the perfusion has some problems with regard to the hydrodynamic properties. Minakawa and associates ${ }^{15}$ performed a streamline analysis of the right axillary artery perfusion in the aortic arch aneurysm model. The flow jet with rapid velocity strikes on the posterior wall of the ascending aorta and enters the ascending aorta with vortical flow that is unexpectedly rapid. This type of nonphysiologic flow causes the detachment of atheroma, with the debris consequently washed away into the left common carotid artery. Therefore, in most cases, we preferred ascending aortic cannulation using a dispersion cannula toward the aortic root. The CPB time and lower body deep hypothermic circulatory arrest times were significantly longer in patients with vs without an atherothrombotic aorta. This might have been because more time is required at surgery to avoid dislodging the debris in such aortas or that patients with an atherothrombotic aorta more often undergo concomitant coronary artery bypass grafting.

Although the overall 30-day mortality was $0 \%$, the hospital mortality in patients with atherothrombotic aorta is likely to be greater $(2.9 \%$ vs $0.7 \%, P=.26)$, and the long-term outcome was significantly limited in our study owing to the preoperative underlying risks. Two patients with an atherothrombotic aorta had 1 episode each of paraparesis and bowel necrosis, both possibly related to embolism. Also noteworthy was the greater incidence of prolonged mechanical ventilation in the group with an atherothrombotic aorta, which might be linked to the greater comorbidity of chronic obstructive pulmonary disease. Taken together, our analysis has confirmed that the cohort of patients with an atherothrombotic aorta is at high risk in terms of both short- and long-term outcomes.

PNDs occurred in 4 patients ( $2.2 \%)$ after surgery. All had leukoaraiosis, but multivariate analysis failed to identify leukoaraiosis as a risk factor. The solitary risk factor for PNDs was CPB time, in accordance with observations from Svensson and associates. ${ }^{16}$ Because they all had undergone concomitant coronary artery bypass grafting with 1.8 bypass grafts on average, all 4 patients had a greater than average $\mathrm{CPB}$ time. Postoperative brain imaging 
demonstrated multiple embolizations in 3 of 4 patients. Dispersion cannulas were not used for these 3 patients whose aortic walls, although severely diseased, did not meet the criteria for having an atherothrombotic aorta. Patient 1 had leukoaraiosis and a history of stroke with hemiplegia. In this patient, postoperative brain imaging showed aggravation of the previous infarction, possibly caused by impaired collateral circulation in the potentially salvageable ischemic penumbra. No patients with an atherothrombotic aorta had PNDs, and an atherothrombotic aorta was not identified as a risk factor on multivariate analysis. We can speculate that improper cannula selection and a longer CPB time caused PNDs in the 4 patients and believe that meticulous selection of the cannulation site and cannula type and complete exclusion of the diseased aorta contributed to avoiding PNDs, even when an atherothrombotic aorta was present.

Despite the apparent reversibility of many symptoms of TNDs, it has been shown that this early manifestation of diffuse cerebral damage is associated with long-term deficits in cognitive function. ${ }^{4,17}$ TNDs are almost certainly related to the cerebral ischemia time. Anoxic cell injury occurs inevitably after 30 minutes of circulatory arrest at $15^{\circ} \mathrm{C}$. TNDs have been believed to correlate with the duration of hypothermic circulatory arrest and probably result from inadequate cerebral protection. Fleck and associates ${ }^{18}$ demonstrated that an increased duration of hypothermic circulatory arrest was associated with increased TND severity. ${ }^{18} \mathrm{Hagl}$ and associates ${ }^{19}$ also showed reduced TND rates after implementation of antegrade cerebral perfusion and determined independent risk factors for TND, including age (OR, 1.06 per year, $P<.001)$, dissection $(\mathrm{OR}$, $2.2, P=.001)$, need for coronary artery bypass grafting (OR, 2.1; $P=.006$ ), or other procedures (OR, 3.4; $P=.023)$, and total cerebral protection time (OR, 1.02 per minute; $P<.001) .{ }^{19}$ Our present study using contemporary MRI demonstrated that risk factors for TND were atherothrombotic aorta, extracranial carotid artery stenosis, more than moderate leukoaraiosis, and CPB time. Most patients with TNDs had a combination of risk factors. Our data suggested that other mechanisms, such as microembolic events caused by aortic wall soft atheroma or hypoperfusion caused by cerebrovascular disorder, or both, might be significant factors underlying TND, even when SCP is used.

Leukoaraiosis reflects chronic ischemic damage to the myelin and axons ${ }^{8}$ and is usually associated with severe arteriosclerosis and arteriolosclerosis. The extent of leukoaraiosis might be a marker for the severity of underlying deep brain hypoperfusion due to arteriosclerosis and arteriolosclerosis. Accordingly, previous reports have shown that in aortic surgery that TNDs are more likely to develop in patients with severe leukoariaosis. ${ }^{20,21}$ Atherosclerotic occlusive disease of the arch vessels can cause a low flow state or atheroembolism, resulting in impaired cerebral circulation, with consequent transient ischemic attacks or stroke. $^{22,23}$ The correlation between the percentage of carotid artery stenosis and the reduction of cerebral perfusion during CPB is known. Warwick and associates $^{24}$ demonstrated that the carotid artery diameter, carotid artery plaque length, and hematocrit, in addition to the percentage of stenosis, should be included in any deliberations concerning carotid artery stenosis and cardiac surgery. Regarding atheroembolism, ulcerated plaque in the common carotid artery can lead to embolic events, particularly when SCP is used for brain protection. Despite the potential advantages of SCP, atherosclerotic occlusive disease of extracranial carotid artery can be a risk factor for TNDs in accordance with our results. The duration of $\mathrm{CPB}$, as a marker of depressed cardiac function and overall reduced cerebral perfusion, can also be a predictor of a poor surgical outcome, particularly in cases in which the CPB times are prolonged. Our present study identified CPB time as a risk factor for TNDs as well as PNDs. However, the potential for interaction of the various risk factors for TNDs has not been thoroughly investigated. We determined the potential role of independent risk factors for TNDs by deriving the $\mathrm{P}_{\mathrm{TND}}$ equation. Our data have demonstrated that atherothrombotic aorta combined with leukoaraiosis, extracranial carotid artery disease, and prolonged CPB time exponentially accelerated the incidence of TNDs, suggesting that patients with the combined risk have a lower threshold for the occurrence of microemboli or hemodynamic shifts during CPB. The probability of TNDs in patients with an atherothrombotic aorta combined with leukoaraiosis and extracranial carotid artery stenosis was as great as $48.0 \%$ at 160 minutes of CPB time. Our analysis has some unique aspects and might allow a more precise calculation of risk, because previous studies have concentrated on the risks of individual features rather than composites. From a surgical point of view, the approach to patients with an atherothrombotic aorta should involve meticulous selection of the arterial cannula type and site, and the CPB time should be shortened by early rewarming just as in our study and elsewhere. ${ }^{3}$ Carotid artery plaque features are also an important consideration when deciding whether to use SCP. In the present study, 2 patients had common carotid artery ulceration with mobile plaque. In these 2 patients, we abandoned the use of SCP in favor of deep hypothermia with RCP, resulting in a satisfactory neurologic outcome. A combination of various techniques should be considered, recognizing the difficulty in recommending a unidirectional approach to brain protection.

\section{Study Limitations}

This was a retrospective study from a single center. The logistic regression analysis for PNDs was limited by the small number of events, although our standardized surgical approach provided satisfactory outcomes. The cerebral 
protection strategy (SCP or RCP) used was not entered into the univariate or multivariate analysis, because RCP was applied to a limited number of patients $(\mathrm{n}=2)$. To investigate the pure effect of an atherothrombotic aorta on PNDs or TNDs, we must apply propensity matching for the comparison. However, such an analysis was not done, because the number of patients with an atherothrombotic aorta was insufficient.

\section{CONCLUSIONS}

Patients with an atherothrombotic aorta have more preoperative comorbidities, which can adversely affect the short- and long-term outcomes, including TNDs. An atherothrombotic aorta combined with leukoaraiosis, extracranial carotid artery stenosis, and prolonged CPB time exponentially increased the incidence of postoperative TNDs in patients undergoing TAR and might, therefore, deserve special attention.

\section{References}

1. Kazui T, Yamashita K, Washiyama N, Terada H, Bashar AH, Suzuki K, et al. Aortic arch replacement using selective cerebral perfusion. Ann Thorac Surg. 2007;83:S796-8.

2. Ogino H, Sasaki H, Minatoya K, Matsuda H, Tanaka H, Watanuki H, et al. Evolving arch surgery using integrated antegrade selective cerebral perfusion: impact of axillary artery perfusion. J Thorac Cardiovasc Surg. 2008;136:641-8.

3. Okada K, Omura A, Kano H, Sakamoto T, Tanaka A, Inoue T, et al. Recent advancements of total aortic arch replacement. J Thorac Cardiovasc Surg. 2011 September 26 [E-pub ahead of print].

4. Ergin MA, Galla JD, Lansman L, Quintana C, Bodian C, Griepp RB. Hypothermic circulatory arrest in operations on the thoracic aorta: determinants of operative mortality and neurologic outcome. J Thorac Cardiovasc Surg. 1994; 107:788-97.

5. Okita Y, Ando M, Minatoya K, Kitamura S, Takamoto S, Nakajima N. Predictive factors for mortality and cerebral complications in arteriosclerotic aneurysm of the aortic arch. Ann Thorac Surg. 1999;67:72-8.

6. Amarenco P, Cohen A, Tzourio C, Bertrand B, Hommel M, Besson G, et al. Atherosclerotic disease of the aortic arch and the risk of ischemic stroke. N Engl J Med. 1994;331:1474-9.

7. Hollier LH, Kazmier FJ, Ochsner J, Bowen JC, Procter CD. "Shaggy" aorta syndrome with atheromatous embolization to visceral vessels. Ann Vasc Surg. 1991; 5:439-44.

8. Schmidt R, Scheltens P, Erkinjuntti T, Pantoni L, Markus HS, Wallin A, et al. White matter lesion progression: a surrogate endpoint for trials in cerebral small-vessel disease. Neurology. 2004;63:139-44.
9. Scheltens P, Barkhof F, Leys D, Pruvo JP, Nauta JJ, Vermersch P, et al. A semiquantitative rating scale for the assessment of signal hyperintensities on magnetic resonance imaging. J Neurol Sci. 1993;114:7-12.

10. The North American Symptomatic Carotid Endarterectomy (NASCET) Collaborators. Beneficial effect of carotid endarterectomy in symptomatic patients with high-grade carotid stenosis. N Engl J Med. 1991;325:445-53.

11. Motomura N, Miyata H, Tsukihara H, Takamoto S. Risk model of thoracic aortic surgery in 4707 cases from a nationwide single-race population through a web-based data entry system: the first report of 30-day and 30-day operative outcome risk models for thoracic aortic surgery. Circulation. 2008;118: S153-9.

12. Bachet J, Guilmet D, Goudot B, Dreyfus GD, Delentdecker P, Brodaty D, et al. Antegrade cerebral perfusion with cold blood: a 13-year experience. Ann Thorac Surg. 1999;67:1874-8.

13. Safi HJ, Miller CC III, Lee TY, Estrera AL. Repair of ascending and transverse aortic arch. J Thorac Cardiovasc Surg. 2011;142:630-3.

14. Fukuda I, Fujimori S, Daitoku K, Yanaoka H, Inamura T. Flow velocity and turbulence in the transverse aorta of a proximally directed aortic cannula: hydrodynamic study in a transparent model. Ann Thorac Surg. 2009;87: 1866-71.

15. Minakawa M, Fukuda I, Inamura T, Yanaoka H, Fukui K, Daitoku K, et al Hydrodynamic evaluation of axillary artery perfusion for normal and diseased aorta. Gen Thorac Cardiovasc Surg. 2008;56:215-21.

16. Svensson LG, Crawford ES, Hess KR, Coselli JS, Raskin S, Shenaq SA, et al Deep hypothermia with circulatory arrest: determinants of stroke and early mortality in 656 patients. J Thorac Cardiovasc Surg. 1993;106:19-28.

17. Reich DL, Uysal S, Sliwinski M, Ergin MA, Kahn RA, Konstadt SN, et al. Neuropsychologic outcome after deep hypothermic circulatory arrest in adults. J Thorac Cardiovasc Surg. 1999;117:156-63.

18. Fleck TM, Czerny M, Hutschala D, Koinig H, Wolner E, Grabenwoger M. The incidence of transient neurologic dysfunction after ascending aortic replacement with circulatory arrest. Ann Thorac Surg. 2003;76:1198-202.

19. Hagl C, Ergin MA, Galla JD, Lansman SL, McCullough JN, Spielvogel D, et al. Neurologic outcome after ascending aorta-aortic arch operations: effect of brain protection technique in high-risk patients. J Thorac Cardiovasc Surg. 2001;121: 1107-21.

20. Lin R, Svensson L, Gupta R, Lytle B, Krieger D. Chronic ischemic cerebral white matter disease is a risk factor for nonfocal neurologic injury after total aortic arch replacement. J Thorac Cardiovasc Surg. 2007;133:1059-65.

21. Morimoto N, Okada K, Uotani K, Kanda F, Okita Y. Leukoaraiosis and hippocampal atrophy predict neurologic outcome in patients who undergo total aortic arch replacement. Ann Thorac Surg. 2009;88:476-81.

22. Chambers BR, Norris JW. Outcome in patients with asymptomatic neck bruits N Engl J Med. 1986;315:860-5.

23. Nicolaides AN, Kakkos SK, Kyriacou E, Griffin M, Sabetai M, Thomas DJ, et al Asymptomatic internal carotid artery stenosis and cerebrovascular risk stratification. J Vasc Surg. 2010;52:1486-96.

24. Warwick R, Sastry P, Fontaine E, Poullis M. Carotid artery diameter, plaque morphology, and hematocrit, in addition to percentage stenosis, predict reduced cerebral perfusion pressure during cardiopulmonary bypass: a mathematical model. J Extra Corpor Technol. 2009;41:92-6. 
TABLE E1. Details of PNDs

\begin{tabular}{lccccccccc}
\hline Pt. no. & Age $(\mathbf{y})$ & Symptoms & Postoperative MRI/CT findings & A & E & L & O & CPB time (min) & Causes \\
\hline 1 & 77 & Hemiplegia & Right-side stroke (aggravation) & No & No & Yes & Yes & 211 & Low perfusion \\
2 & 86 & Hemiplegia & Left- and right-side stroke (multiple) & No & No & Yes & No & 170 & Embolic \\
3 & 78 & Hemiplegia & Left- and right-side stroke (multiple) & No & No & Yes & No & 210 & Embolic \\
4 & 78 & Hemiplegia & Left- and right-side stroke (multiple) & No & No & Yes & No & 368 & Embolic \\
\hline
\end{tabular}

$\overline{P N D s}$, Permanent neurologic deficits; Pt. no., patient number; $M R I$, magnetic resonance imaging; $C T$, computed tomography; $A$, atherothrombotic aorta; $E$, extracranial carotid artery stenosis; $L$, leukoaraiosis; $O$, old cerebral infarction; $C P B$, cardiopulmonary bypass.

TABLE E2. Additional postoperative complications

\begin{tabular}{lllll}
\hline & & \multicolumn{3}{c}{ Atherothrombosis } \\
\cline { 3 - 4 } \multicolumn{1}{c}{ Variable } & Total & \multicolumn{1}{c}{ Yes } & No & P value \\
\hline Paraparesis (temporary) & $1(0.56)$ & $1(2.9)$ & $0(0)$ & $.04 *$ \\
Mediastinitis & $3(1.7)$ & $0(0)$ & $3(2.1)$ & .4 \\
$\begin{array}{l}\text { Prolonged ventilation } \\
\quad(\geq 48 \text { h) }\end{array}$ & $23(12.8)$ & $8(23.5)$ & $15(10.3)$ & $.04 *$ \\
Renal failure & & & & \\
Gastrointestinal tract (necrosis) & $1(0.56)$ & $1(2.9)$ & $0(0)$ & $.04 *$ \\
\hline
\end{tabular}

Data presented as numbers, with percentages in parentheses. *Significant difference at $P<.05$ with and without atherothrombosis. 